\title{
Tierwohl in der Landwirtschaft
}

\author{
Matthias Gauly
}

Wie kaum eine andere Branche steht die Landwirtschaft in vielen Ländern Europas im Fokus der öffentlichen Aufmerksamkeit. Vor allem die moderne Nutztierhaltung sieht sich mit verschiedenen Vorwürfen konfrontiert. Im Wesentlichen sind dies Fragen zu Tierwohl und Tiergesundheit, zu den Effekten intensiver Wirtschaftsweisen auf Umwelt und Natur sowie zu negativen Auswirkungen der Produkte auf die menschliche Gesundheit (u. a. Krankheitsresistenz). Zum Synonym für eine wenig tiergerechte Haltung ist der Begriff „Massentierhaltung“ geworden. Er hat sich etabliert und ruft eindeutig negative Assoziationen hervor (Gesa Busch et al. 2013). Dies steht teilweise im krassen Widerspruch zur Selbsteinschätzung der Branche. In Deutschland hat sich die Landwirtschaft selbst vor einem Jahrzehnt noch als eine der wichtigsten Branchen im Lande und als bedeutende $\mathrm{Zu}$ kunftsbranche eingeschätzt (vgl. DLG Wintertagungen 2009 und 2010). Dabei ist die Zukunft einer Branche sehr wesentlich auch von deren Akzeptanz in der Bevölkerung abhängig. Der Wissenschaftliche Beirat für Agrarpolitik beim deutschen Bundesministerium für Ernährung und Landwirtschaft (WBA) sieht in seinem Gutachten vom März 2015 über „Wege zu einer gesellschaftlich akzeptierten Nutztierhaltung“ genau diese nicht mehr als gegeben an und postuliert auch deswegen die Notwendigkeit eines grundlegenden Wandels der Nutztierhaltung (Harald Grethe et al. 2015).

Die Intensivierung der Nutztierhaltung erfolgte in sehr starkem Maße nach den beiden Weltkriegen. Primäres Ziel der Erzeugung war die rasche Bereitstellung tierischer Produkte zu niedrigen Preisen. Die Treiber der Weiterentwicklungen der tierischen Haltungssysteme waren dementsprechend vor allem Aspekte der Ökonomie (ökonomische Nachhaltigkeit), der Ressourceneffizienz (Mitteleinsatz pro erzeugtes Produkt) sowie, etwas später, des Arbeitsschutzes. Heute wird die Entwicklung der Systeme und Haltungsverfahren zusätzlich von gesetzlichen Vorgaben (u. a. Tierschutzgesetz, Tierschutz-Nutztierhaltungsverordnung), Fragen der Tiergesundheit und des Tierschutzes, des Ressourcen- sowie Umweltschutzes bewegt. Während in Deutschland bei der Haltung von Schweinen (u. a. Anpassung der Gruppenhaltung im Wartebereich der Sauen) und Geflügel (u. a. Ver- 
bot der konventionellen Käfighaltung bei Legehennen) eine starke Orientierung an den gesetzlichen Mindestvorgaben (Verordnung zum Schutz landwirtschaftlicher Nutztiere und anderer zur Erzeugung tierischer Produkte gehaltener Tiere bei ihrer Haltung) erfolgt, ist dies im Milchviehbereich nicht der Fall. Hier gibt es bisher keine gesetzlich verbindlichen Vorgaben.

Insgesamt waren Aspekte des Tierwohls sehr lange für die Gestaltung von Haltungsverfahren von eher untergeordneter bzw. indirekter Bedeutung. Dies auch, weil Tierwohl nach Auffassung von Teilen der Praxis und Wissenschaft mit anderen Zielen der Erzeugung, z. B. der Wirtschaftlichkeit, häufig im Konflikt steht. Unterstellte Korrelationen sind allerdings teilweise entweder nicht oder in wesentlich geringerem Maße vorhanden. Es wird dennoch häufig als Standardargument gegen Bestrebungen der Verbesserung von Haltungsverfahren vorgebracht.

Im folgenden Beitrag werden zunächst die Begriffe Tierwohl, Tiergesundheit und Tierschutz definiert und dann die Entwicklung der Nutztierhaltung exemplarisch am Beispiel Deutschlands dargestellt. Anschließend wird die aktuelle Situation des Tierwohls und der Tiergesundheit in einigen Bereichen der Nutztierhaltung erläutert. Darauf aufbauend werden schlussendlich Wege zu einer Verbesserung der Tierwohlsituation aufgezeigt.

\section{Was versteht man unter Tierwohl, Tiergesundheit und Tierschutz und wie} kann man diese bewerten?

Die europäische Tierschutzgesetzgebung gibt fast einheitlich als Ziel vor, dass das Leben und Wohlbefinden von Tieren geschützt und ihnen ohne „vernünftigen“ Grund keine Schmerzen, Leiden oder Schäden zugefügt werden sollen. Entsprechend schlägt Ute Knierim (2010) vor, die wesentlichen Zielgrößen der Tierschutzgesetzgebung (Abwesenheit von Schmerzen, Leiden und Schäden sowie Sicherung von Wohlbefinden) unter dem Begriff Wohlergehen zusammenzufassen. Dieser Begriff beinhaltet somit, anders als Wohlbefinden, nicht nur psychische, sondern auch körperliche Aspekte. Dies trifft auch auf die in jüngster Zeit häufiger gebräuchliche Bezeichnung „Tierwohl“ zu. Die WHO hat bereits 1946 für die menschliche Gesundheit festgestellt, dass sie ein „Zustand vollständigen körperlichen, geistigen und sozialen Wohlbefindens, und nicht nur die Abwesenheit von Krankheit und Gebrechen" ist. Später definierte die New Common Animal Health Strategy der EU (2007) darauf aufbauend auch, dass Tiergesundheit nicht nur die Freiheit von Krankheiten ist, sondern das Wohlergehen der 
Tiere mit einbezieht. Ein weiterer Begriff ist der der „Tiergerechtheit“. Dieser drückt nach Ute Knierim (2001) aus, in welchem Maß es den Tieren gelingt, unter den zu beurteilenden Umweltbedingungen Schmerzen, Leiden und Schäden zu vermeiden sowie ein gutes Wohlbefinden zu erreichen. Sie kann, wie auch die Tiergesundheit (Aaron Antonovsky 1996), entlang einem Kontinuum von wenig bis sehr tiergerecht reichen. Tiergesundheit bedeutet nach Diedrich Smidt (1996), dass Tiere morphologischfunktionell unversehrt sind und ihre Fähigkeit zur physiologischen Kompensation haltungs- und leistungsbedingter Belastungen unbeeinträchtigt ist. In diesem Zusammenhang bekommt die Beurteilung der „Nutzungsdauer" von Tieren eine besondere Bedeutung für die Bewertung der Tiergerechtheit von Haltungsverfahren. Die kurze Lebensdauer von Tieren und deren meist erkrankungsbedingte Abgänge (z. B. bei Milchkühen) weisen sehr stark auf eine „Überforderung“ derselben hin.

In der Vergangenheit wurden für die Einschätzung des Tierwohlniveaus von Haltungssystemen praktisch ausschließlich die Umweltbedingungen wie Flächenangebot oder Bodengestaltung herangezogen („Tierschutz mit dem Zollstock"). Der große Nachteil solcher haltungs- und managementbezogenen Messgrößen liegt in ihrem häufig geringen Erklärungswert für das tatsächliche Wohlergehen von Tieren. Zunehmend wird daher anerkannt, dass alleine auf der Basis von Umweltbedingungen und Leistungsmerkmalen die Lebensqualität der Tiere nicht korrekt beurteilt werden kann. Die Kombination mit tierbezogenen Messgrößen der Gesundheit (z. B. Hautverletzungen, Erkrankungsfrequenzen) und des Verhaltens führen grundsätzlich zu gültigeren Aussagen. Gleichzeitig bringt deren Erfassung aber erhebliche Herausforderungen mit sich, z. B. bezüglich Praktikabilität, standardisierter Erfassung oder der Verallgemeinerbarkeit einzelner Parameter (Daniel Gieseke et al. 2014). Neben der zunehmenden Würdigung der Bedeutung tierbezogener Tierwohlindikatoren rückt auch die Sicherung von Wohlbefinden, was das Erleben positiver Emotionen einschließt, in den Fokus der Betrachtungen. Letztere sind kurzfristige, angenehme Empfindungen, die sich aus derzeitiger wissenschaftlicher Sicht sehr positiv auf das Wohlergehen von Tieren auswirken (Alain Boissy et al. 2007). Es darf angenommen werden, dass mit der Erforschung der Fähigkeit der Wahrnehmung von Emotionen an Nutztieren sich auch die grundsätzliche Einstellung zur Behandlung und „Nutzung“ von Tieren ändern wird. 


\section{Die Entwicklung der Nutztierhaltung - Beispiel Deutschland}

Die Entwicklung der Tierhaltung im Hinblick auf Betriebszahlen und Bestandsgrößen sind beispielhaft für Deutschland in den Tabellen 1 und 2 dargestellt. Einem kontinuierlichen Rückgang der landwirtschaftlichen Betriebe steht, wie in allen anderen europäischen Ländern, ein stetiges Größenwachstum entgegen. Wenngleich entsprechende Konzentrationsprozesse in allen Wirtschaftsbereichen stattfinden, werden sie in der Landwirtschaft besonders kritisch gesehen, da ein Zusammenhang mit Tierwohl und Tiergesundheit unterstellt wird. Der Begriff „Massentierhaltung“ hat sich etabliert. In der gesamten Bevölkerung ist, wenn auch auf unterschiedlichem Niveau, eine ablehnende Haltung gegenüber „Massentierhaltung" und damit verbundenen Produktionsmethoden festzustellen (Gesa Busch et al. 2013), obwohl kaum wissenschaftlich belastbare Erkenntnisse über die Zusammenhänge von Bestandsgrößen und Tierwohl vorliegen (Matthias Gauly 2015) (siehe Kapitel 3.5: Bestandsgrößenentwicklung und Tierwohl).

\begin{tabular}{cccc}
\hline Betrieb & 2000 & 2019 & $\begin{array}{c}\text { Jährlicher Rück- } \\
\text { gang in Prozent }\end{array}$ \\
\hline $\begin{array}{c}\text { Sauen } \\
\begin{array}{c}\text { Mast- } \\
\text { schweine }\end{array}\end{array}$ & 77.000 & 7.200 & $-4,5$ \\
Milchkühe & 139.000 & 17.900 & $-4,0$ \\
Legehennen & 2.500 & 59.925 & $-3,0$ \\
Mast- & 1000 Plätze $)$ & $(>3000 \text { Plätze })^{1}$ & $-3,5$ \\
hähnchen & 11.300 & $3.330^{2}$ & $-4,4$ \\
\hline
\end{tabular}

Tabelle 1: Betriebszahlenentwicklung in Deutschland zwischen 2000 und 2019 (ZMP 2003, 2012; Destatis 2011, 2020; ${ }^{1}$ Stand 2013, ${ }^{2}$ Stand 2016).

\begin{tabular}{cccc}
\hline Tierzahl pro Betrieb & 2000 & 2019 & $\begin{array}{c}\text { Jährlicher Zuwachs } \\
\text { in Prozent }\end{array}$ \\
\hline Mastschweine & 294 & 655 & $+6,5$ \\
Milchkühe & 31 & 67 & $+6,1$ \\
Masthähnchen & 4.542 & $14.900^{1}$ & $+22,8$ \\
\hline
\end{tabular}

Tabelle 2: Bestandsgrößenentwicklung in Deutschland zwischen 2000 und 2019 (ZMP 2003, 2012; Destatis 2011, 2020; ${ }^{1}$ Stand 2010). 
Weitere Veränderungen sind in der steigenden regionalen Konzentration der Tierhaltung zu sehen. Zu den Regionen höchster Konzentration gehören z. B. in Deutschland Vechta und Cloppenburg. Während z. B. 2010 im Bundesdurchschnitt der Schweinebesatz bei 165 Tieren pro 100 ha lag, war er in den genannten Regionen mit 1.674 bzw. 1.327 Tieren um ein Vielfaches höher. Dabei zeigen sich in verschiedenen Bereichen im Vergleich von Betrieben in intensiven und extensiven Regionen durchaus Vorteile der Konzentration. So belegen Ludwig Theuvsen und Mark Deimel (2011), dass in Betrieben aus Intensivregionen Tierverlustraten niedriger sowie umgekehrt die tierischen Leistungen und damit auch die Bioeffizienz signifikant besser sind. Die Gründe dafür sind vielfältige (u. a. bessere Verfügbarkeit von Beratungs- und Betreuungseinrichtungen). Nicht vernachlässigt werden dürfen allerdings die negativen Effekte regionaler Konzentration der Nutztierhaltung. So sind z. B. die Zusammenhänge zwischen der räumlichen Konzentration der Tierhaltung, hohen Stickstoff-Salden, hohen Belastungen des Grund- und Oberflächenwassers mit Nitrat sowie hohen Ammoniakemissionen zu nennen. So weist der deutsche Sachverständigenrat für Umweltfragen im Sondergutachten „Stickstoff: Lösungsstrategien für ein drängendes Umweltproblem“ (2015) nachdrücklich auf die Überschreitung des critical loads an Stickstoff in Intensivregionen hin. Kann dieses Problem z. B. durch verschärfte Düngeregelungen (und deren konsequente Einhaltung) nicht gelöst werden, ist über regionale Bestandsobergrenzen nach niederländischem Muster nachzudenken (Harald Grethe et al. 2015). Hinzu kommt, dass in Intensivregionen aufgrund rechtlicher Rahmenbedingungen ( $u$. a. Umweltregelungen, Abstandsregeln) bestimmte Haltungsverfahren (z. B. Auslauf, Freiland) nicht zulassungsfähig sind. An dieser Stelle werden die möglichen Effekte sowie Zielkonflikte zwischen regionaler Konzentration, Umweltbelastung und Tierwohl deutlich.

\section{Verschiedene Problemfelder der Nutztierhaltung}

\subsection{Das Milchvieh}

Das Milchvieh schneidet hinsichtlich des Tierwohls sowie der Tiergesundheit in der Wahrnehmung durch den Verbraucher im Vergleich zu anderen Tierhaltungssystemen überdurchschnittlich gut ab (Sarah Kühl et al. 2014). Die Milchviehhaltung rangiert auf Platz eins vor der Schafhaltung. Kälber- und Geflügelmast belegen die letzten Plätze bei Befragungen (Reimar von Alvensleben 2003). Das positive Abschneiden in der öffentlichen 
Wahrnehmung hat vermutlich verschiedene Gründe. Dazu gehört zweifelsohne auch, dass die Sichtbarkeit der Tiere bzw. Betriebe in der Regel gegeben ist. Die Bestände sind nicht, im Gegensatz zu Schweine- und Geflügelbetrieben, aus Hygienegründen abgeriegelt, sondern prinzipiell zugänglich. Dies schafft offenbar ein gewisses Grundvertrauen. Aus Sicht des Tierwohls und des Verbrauchers sehr positiv bewertet wird auch die primär aus ökonomischen Gründen erfolgende Entwicklung von der Anbinde- zur Laufstallhaltung (Matthias Gauly 2015). Als ein besonderer „Imageförderer" erweist sich bei Verbraucherbefragungen der Faktor Weidehaltung (Sarah Kühl et al. 2014). Die Werbung für Milch und Milchprodukte hat dieses Image wesentlich mitgeprägt. Tatsächlich hängen das Wohl und die Gesundheit von Tieren auch davon ab, ob sie ihre natürlichen Verhaltensweisen ausleben können. Dies ist bei Weidehaltung mehr gegeben als bei reiner Stallhaltung (Linda Armbrecht et al. 2015). Demgegenüber ist aber in ganz Europa ein Rückgang der Weidehaltung zu verzeichnen, wie eine umfangreiche Studie über die erwartete Entwicklung der Milcherzeugung in verschiedenen europäischen Ländern, durchgeführt vom LEI-Institut in Wageningen, feststellte (Joan Reijs et al. 2013). Bis zum Jahr 2025 wird danach für die Niederlande ein deutlicher Rückgang der Milch aus Vollweidehaltung von derzeit 35 auf 9 Prozent erwartet. Noch gravierender ist die Prognose für Deutschland. Mit der stärkeren Entwicklung hin zur reinen Stallhaltung muss eine Reduktion des Tierwohls (u. a. Ausleben von Emotionen) sowie ein nicht unerhebliches Imagerisiko für die Milchbranche befürchtet werden. Die Umstellung erfolgt dennoch, da die ganzjährige Stallhaltung insbesondere von größeren Betrieben als rentabler und einfacher angesehen wird, da ressourceneffizienter und mit weniger Risiko behaftet. Dabei werden Entscheidungen für oder gegen ein Haltungssystem auf Betriebsebene häufig sehr stark auf der Basis der erwarteten Jahresleistung pro Kuh getroffen (Vergleichbares gilt auch für andere Tierarten). Ob diese Zielgröße allerdings immer mit Produktionseffizienz und Wirtschaftlichkeit korreliert, ist häufig unklar. Unberücksichtigt bleiben bei den Entscheidungen zum Betrieb eines Haltungssystems meist auch Bestandsergänzungskosten (Peter Thomet/Valérie Piccand 2011) sowie gesellschaftlich bedeutende Zusatzleistungen (u. a. Landschaftsbild, Tierwohl, Biodiversität).

Die Validierung der aktuellen Situation der Tiergesundheit kann bei allen Tierarten anhand verschiedener Parameter erfolgen. Dazu gehören z. B. die Erkrankungsfrequenzen bzw. die Zahl tierärztlicher Behandlungen in einem Bestand sowie die Nutzungsdauer von Tieren. In Tabelle 3 sind die Erkrankungshäufigkeit sowie das Leistungsniveau von Milchkühen aus Testherden in Brandenburg dargestellt (Margret Roffeis/Benno 
Waurich 2013). Neben dem Zusammenhang zur Leistung fällt vor allem der im Laufe einer Laktation geringe Anteil unbehandelter (d. h. gesunder) Kühe negativ auf. Praktisch jedes Tier musste mindestens einmal tierärztlich behandelt werden. Dies spricht sehr deutlich für eine schlechte Gesundheitssituation der Bestände und unterstreicht den Handlungsbedarf. $\mathrm{Zu}$ den häufigsten Abgangsursachen beim Milchvieh zählen Fruchtbarkeitsstörungen sowie Erkrankungen des Euters und Bewegungsapparates.

\begin{tabular}{ccccc}
\hline & \multicolumn{2}{c}{ Erkrankungshäufigkeit je Kuh und Jahr } & $\begin{array}{c}\text { Anteil } \\
\text { gesunder Tiere }\end{array}$ \\
\hline $\begin{array}{c}\text { Leistungsgruppe } \\
\text { nach 100-Tage } \\
\text { Milch-kg }\end{array}$ & Gesamt & Euter & $\begin{array}{c}\text { Bewegungs- } \\
\text { apparat }\end{array}$ & Prozent \\
\hline $\begin{array}{c}<000 \\
3000-3500\end{array}$ & 2,98 & 0,52 & 0,91 & 13,0 \\
$3500-4000$ & 3,10 & 0,53 & 0,94 & 11,9 \\
$>4000$ & 3,48 & 0,60 & 0,93 & 10,6 \\
\hline
\end{tabular}

Tabelle 3: Erkrankungshäufigkeit und Leistungsniveau von Milchkühen aus Testherden in Brandenburg (Margret Roffeis/Benno Waurich 2013).

Ein weiterer wichtiger Indikator zur Bewertung von Haltungssystemen ist die Nutzungsdauer eines Tieres. In den Jahren von 2004 bis 2013 hat sich zwar die Nutzungsdauer (Länge des produktiven Lebens in Monaten von erster Kalbung bis zum Abgang) aufgrund intensiver züchterischer Bemühungen bei Milchkühen bei einzelnen Zuchtverbänden (Beispiel Brandenburg $+2,2$ Monate) erhöht (bei einigen ist sie gleich geblieben, Beispiel Weser-Ems), lag aber dennoch nach wie vor auf einem sehr unbefriedigend niedrigen Niveau (Abb. 1). Das durchschnittliche Abgangsalter der Kühe liegt bei 5,4 Jahren, also bei etwas mehr als zwei Laktationen. Dies ist auch aus wirtschaftlicher Sicht eindeutig als zu gering zu bewerten (Anke Römer 2011) und unterstreicht deutlich Probleme bei der Tiergesundheit.

Die dargestellten Probleme werden mittlerweile auch von der Praxis erkannt und Lösungskonzepte umgesetzt. Verschiedene Milchgenossenschaften bzw. milchverarbeitende Unternehmen haben in den vergangenen Jahren beispielsweise sogenannte „Nachhaltigkeitsstrategien“ vorgelegt, die auch Aspekte des Tierwohls bzw. der Tiergesundheit umfassen. Der Deutsche Milchkontor (DMK), eines der größten deutschen Molkereiunternehmen, verfolgt beispielsweise nach eigenen Angaben mit der im Jahr 2013 veröffentlichten Nachhaltigkeitsstrategie DMK 2020 auch einen umfassen- 


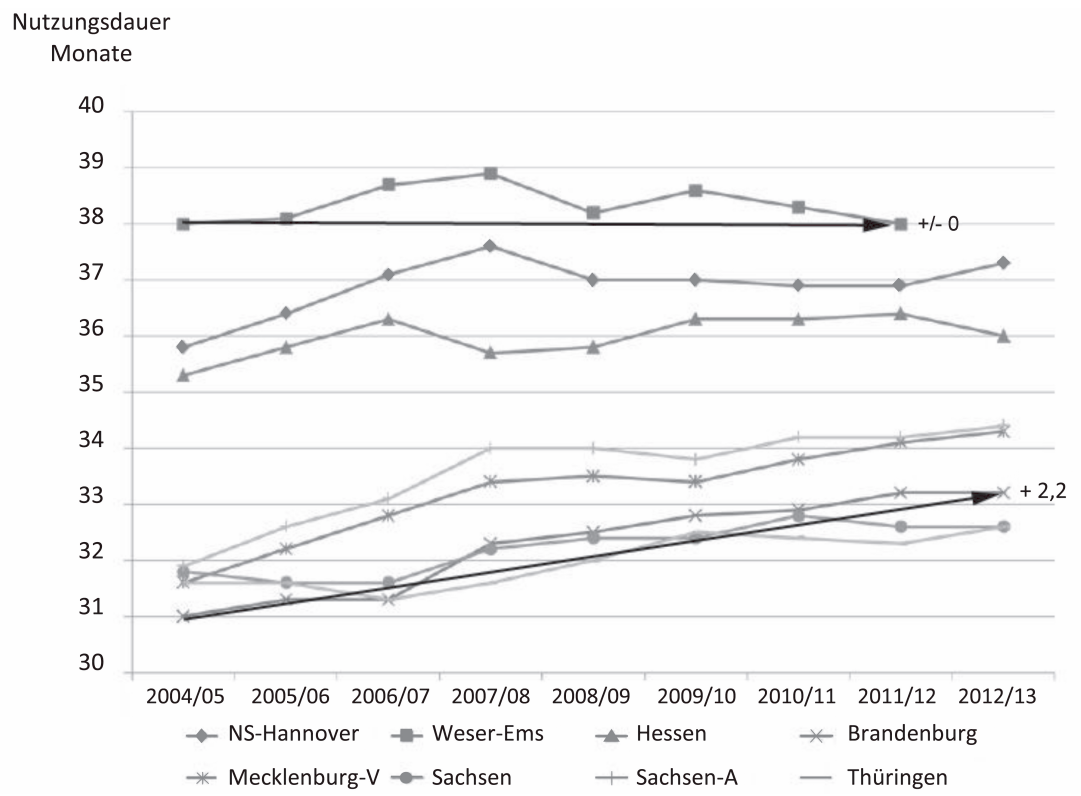

Abb. 1: Entwicklung der Nutzungsdauer (Länge des produktiven Lebens in Monaten von erster Kalbung bis zum Abgang) in verschiedenen Zuchtverbänden auf der phänotypischen Ebene (Zusammenstellung auf Basis der Jahresberichte des VIT, Verden).

den Ansatz zu Tierwohl und Umweltschutz. Dabei wurden Aspekte des Kuhkomforts, der Tiergesundheit, des Futteranbaus und der Fütterung sowie der Milchqualität integriert. Seit 2015 werden alle Milcherzeuger anhand von 148 Kennzahlen (u. a. Haltungssystem) jährlich überprüft. Das DMK bietet seit 2016 seinen Milcherzeugern ein Bonusprogramm zur Förderung nachhaltiger Milcherzeugung. Mit Beginn des Jahres 2020 ist eine Programmanpassung erfolgt. Mögliche Entwicklungen werden nicht transparent dargestellt. Der Aufgabe einer umfassenden Darstellung der Nachhaltigkeit der Milcherzeugung hat sich auch das Thünen-Institut zusammen mit dem QM-Milch e. V., dem Projektbüro Land und Markt und weiteren Akteuren der Milchwirtschaft im Jahr 2017 gestellt und das „Nachhaltigkeitsmodul Milch“ erarbeitet. ${ }^{1}$ Ebenfalls eingebunden ist der Landeskontrollverband Nordrhein-Westfalen (Datenbank). Es nehmen 34

1 Siehe dazu die Webpage https://www.qm-milch.de/nachhaltigkeit (abgerufen am 17.04.2020). 
Molkereien unterschiedlicher Größe und Ausrichtung an dem Pilotprojekt teil. Bestandteile sind ein Fragebogen zur Erhebung von Nachhaltigkeitskriterien in den Milchviehbetrieben, ein Bericht mit Erläuterungen und Bewertungsoptionen sowie ein Online-Eingabetool des Fragebogens und eine zentrale Datenbank. Das „Nachhaltigkeitsmodul Milch“ baut unmittelbar auf zwei Vorläuferprojekten in Niedersachsen und Schleswig-Holstein auf. Das Projekt wird von den Verantwortlichen als ein Auftakt und Instrument für einen kontinuierlichen Lern- und Entwicklungsprozess gesehen. Darauf aufbauend soll ein zukunftsfähiges Konzept erarbeitet werden, das auch Elemente des Tierwohls umfasst (Birthe Lassen et al. 2019).

\subsection{Die Mastschweinehaltung}

Nach Angaben des Deutschen Statistischen Bundesamtes standen bereits 2010 mehr als 90 Prozent aller Schweine in Deutschland auf perforierten Böden (WBA 2015, 92). Diese Form der Haltung wird wesentlich für das Auftreten von Etho- (Verhaltensstörungen bzw. Abweichungen vom Normalverhalten) und Technopathien (durch Haltungsumwelt verursachte Erkrankungen) verantwortlich gemacht. Letzteres sind vor allem Erkrankungen im Bereich der Gliedmaßen sowie der Atemwegsorgane. Die komplex verursachte Verhaltensstörung Schwanzbeißen tritt vor allem in solchen konventionellen Schweinehaltungen und in deutlich geringerem Ausmaß in alternativen Systemen wie der Freilandhaltung und der ökologischen Schweinehaltung auf (Lília Thays Sonoda et al. 2013). Trotz des grundsätzlichen Verbots durch EU-Recht und nationale Tierschutzgesetzgebungen wird deshalb das Kürzen des Schweineschwanzes zur Kannibalismusvorbeuge nach wie vor umfassend durchgeführt (siehe Kapitel 3.4).

Sophie Meyer-Hamme et al. (2016) stellten bei Untersuchungen nach dem Welfare Quality® Protokoll (WQP) in konventionellen Schweinemastbetrieben fest, dass das durchschnittliche Tierwohlniveau als niedrig bis sehr niedrig einzustufen ist. Dies betraf vor allem die Faktoren Verhalten und Gesundheit der Tiere. Als Problembereiche wiesen die Ergebnisse auf die Kriterien Überbelegung und Wasserangebot und damit tierschutzrelevante Punkte hin. So waren beim Kriterium „Bewegungsfreiheit“ unter Berücksichtigung der deutschen Tierschutz-Nutztierhaltungsverordnung von 2006 rund 40 Prozent der Buchten überbelegt, d. h. verstießen gegen gesetzliche Vorgaben. Davon waren 92 Prozent der Betriebe betroffen. Auch wurden hinsichtlich des Kriteriums „Abwesenheit von Durst“ teilweise erhebliche Mängel bei der Wasserversorgung festgestellt. 


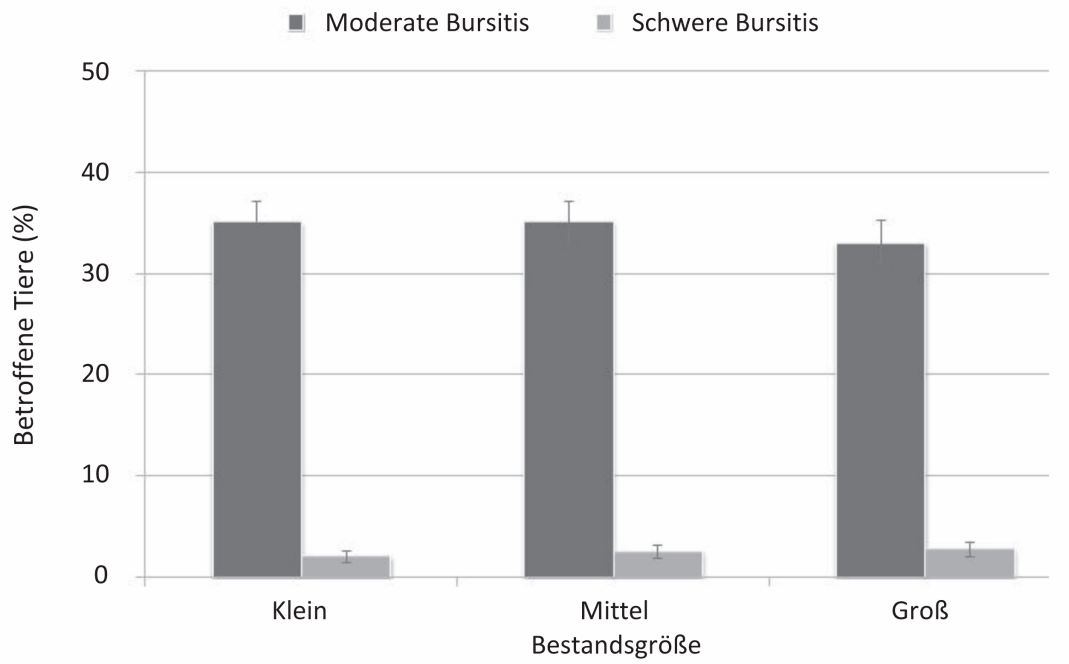

Abb. 2: Prävalenz von Hilfsschleimbeuteln (Bursitis) bei Mastschweinen aus Spaltenbodenhaltungen (92 Prozent Voll-, 8 Prozent Teilspalten) (60 Betriebe; $\mathrm{n}=$ 8.214 Tiere) (Sophie Meyer-Hamme et al. 2016).

Die dargestellten Probleme werden in verschiedenen Brancheninitiativen (ähnlich dem Milchviehbereich) mit unterschiedlichen Konzepten bearbeitet. So setzt die ITW - Initiative Tierwohl ${ }^{2}$ auf die Einführung und Nutzung eines Labels. Die ITW zeichnet die Geflügel- und Schweinefleischprodukte aus, die von einem Betrieb stammen, der an der Initiative teilnimmt und deren Tierwohlkriterien umsetzt. Letztere liegen allerdings nur geringfügig über den gesetzlich vorgegebenen Standards. An der vom Handel unterstützten Initiative nehmen 2020 ca. 7.000 Betriebe teil.

\subsection{Leistung, Tierwohl und Tiergesundheit}

Der Bereich der Tiergesundheit muss über alle Tierarten hinweg als kritisch gesehen bzw. als signifikant verbesserungswürdig benannt werden (Matthias Gauly 2015). Als Gründe für die unbefriedigende Situation können zwei Hypothesen genannt werden: Zum einen sind für viele Betriebe (bzw. Betriebsleiter) die hohen genetisch veranlagten Leistungen bei allen

2 Siehe dazu die Homepage der ITW: https://initiative-tierwohl.de (abgerufen am 17.04.2020). 
Tierarten nicht oder nur sehr schwer beherrschbar. Zum anderen muss festgestellt werden, dass für einige Veränderungen (z. B. die Leistungszuwächse) die teilweise in der Praxis eingesetzten Stallsysteme nicht ausgelegt sind. Der Kurzstand der Milchkuh ist dafür ein Beispiel. Die Selektion auf hohe Milchleistung hat zu größeren Tieren geführt (höhere Futteraufnahme). Solche Tiere finden heute keinen ausreichenden Platz mehr in diesem traditionellen Haltungssystem (Kurzstand). Hohe Frequenzen an Technopathien sind die Folge. Lange Abschreibungszeiträume für Stallungen verhindern eine kontinuierliche Anpassung an die extrem schnellen Entwicklungen. D. h., die Zuchtfortschritte erfolgen rascher als haltungstechnische Anpassungen. Als weiteres Beispiel sei die Entwicklung der Ferkelzahlen pro Sau und das damit verbundene mangelnde Platzangebot zu nennen. Eine Anpassung an die in kurzer Zeit rasant steigenden Fruchtbarkeitsleistungen war nicht möglich. Platzmangel und Überbelegungen im Abferkel- und Flatdeckbereich sind die Folge. Aus ökonomischen Gründen können Landwirte allerdings nicht auf die Nutzung der „produktiven“, für einige Stallsysteme ungeeigneten Genotypen verzichten. Die Konsequenz sind Probleme des Tierwohls. Es ist anzunehmen, dass die neuen Möglichkeiten der genomischen Selektion das Problem weiter verschärfen werden. Die Zuchtverbände und Wissenschaftler müssen diese Entwicklung in Zukunft stärker in ihrer Verantwortung für die Praxis berücksichtigen (Matthias Gauly 2015).

Auch bei Legehennen hat ein extremer, im Wesentlichen züchterisch bedingter Leistungszuwachs stattgefunden. Die Folge ist in Kombination mit fehlerhaften Haltungselementen (z. B. Anflugstangen) fatal. Ca. 50 Prozent aller Legehennen erleiden nach britischen Untersuchungen (Victoria Sandilands 2011) im Laufe einer Legeperiode mindestens einen Knochenbruch. Die Prävalenz war bei den Untersuchungen nicht signifikant unterschiedlich zwischen den zu diesem Zeitpunkt üblichen Haltungssystemen (Boden-, Freiland- und Käfighaltung). Unterscheiden ließ sich jedoch der Zeitpunkt der Entstehung der Frakturen. Sie wies zumindest teilweise auf Mängel der Systeme und des Managements hin (z. B. mehr frische Fakturen bei Tieren aus konventioneller Haltung als bei Tieren aus Boden- und Freilandhaltung). Die Systemmängel müssten bereits vor der Marktreife und dem Verkauf des Produktes (der Haltungseinrichtung bzw. des Haltungssystems) an Landwirte behoben sein! Vor diesem Hintergrund ist es nicht ganz verständlich, warum sich die Berufsverbände in einigen Ländern so sehr gegen die Einführung eines Prüf- und Zulassungsverfahrens von Stalleinrichtungen und Systemen wehren. 


\begin{tabular}{lcccc}
\hline & $\begin{array}{c}\text { konventio- } \\
\text { neller Käfig }\end{array}$ & $\begin{array}{c}\text { ausgestalteter } \\
\text { Käfig }\end{array}$ & Freiland & Boden \\
\hline $\begin{array}{l}\text { Betriebe (n = jeweils } \\
100 \text { Hennen/Betrieb) }\end{array}$ & 8 & 4 & 9 & 3 \\
\hline $\begin{array}{l}\text { Prozent der Tiere } \\
\text { mit: }\end{array}$ & 23 & 13 & 9 & 15 \\
$\begin{array}{l}\text { frischer Fraktur } \\
\text { alter Fraktur }\end{array}$ & 26 & 30 & 45 & 53 \\
\hline $\begin{array}{l}\text { Total Prozent der } \\
\text { Tiere mit Fraktur }\end{array}$ & 49 & 43 & 54 & 68 \\
\hline
\end{tabular}

Tabelle 4: Legehennen: Knochenbrüche in Abhängigkeit vom Haltungsverfahren (Victoria Sandilands 2011).

\subsection{Eingriffe am Tier und Tierwohl}

Bei der Diskussion um nicht-kurative Eingriffe an landwirtschaftlichen Nutztieren steht je nach Eingriff die grundsätzliche Frage nach dessen Unerlässlichkeit und des Zufügens von Schmerzen und Leiden im Raum. Nicht-kurative Eingriffe sind operative und schmerzhafte Manipulationen an Tieren, die dem Zweck dienen, das Management zu erleichtern, Haltungseinrichtungen zu schonen, das Risiko für Verletzungen von Tieren und Menschen zu vermindern (z. B. Enthornung von Rindern, Kupieren der Schwänze beim Schwein, Kupieren der Schnäbel bei Legehennen) und/oder die Produktqualitäten zu beeinflussen (z. B. Kastration von männlichen Mastschweinen). In den meisten europäischen Tierschutzgesetzgebungen sind nicht-kurative Eingriffe bei Wirbeltieren, die mit der Zerstörung von Gewebe einhergehen, grundsätzlich verboten („Amputationsverbot“). Die Gesetzgebung verfolgt also grundsätzlich das Ziel, die Integrität der Tiere zu schützen. Gleichzeitig sind grundsätzlich Schmerzausschaltung und -behandlung bei notwendigen schmerzhaften Eingriffen vorgeschrieben. Es gibt allerdings eine Reihe von Ausnahmetatbeständen sowohl vom "Amputationsverbot" als auch von der Betäubungspflicht, insbesondere für die landwirtschaftliche Nutztierhaltung. Dazu gehören prinzipiell, länderabhängig, unter bestimmten Altersbeschränkungen neben der Tierkennzeichnung durch Ohrmarken, Tätowierung, Chip oder beim Pferd Brand, z. B. das Zerstören der Hornanlagen bei Rindern, das Kastrieren von Ebern, Bullen und Böcken, das Schwanzkürzen bei Ferkeln, Lämmern und Kälbern und das Schnabelkürzen bei Nutzgeflügel. Die genannten Eingriffe außer der Kastration dürfen allerdings nach Gesetzeslage in der Regel (länderspezifische Unterschiede existieren) nur durchgeführt 
werden, wenn „der Eingriff im Einzelfall für die vorgesehene Nutzung des Tieres zu dessen Schutz oder zum Schutz anderer Tiere unerlässlich ist“". Diese Bestimmungen werden allerdings in der Praxis überwiegend nicht ernsthaft berücksichtigt, denn das würde bedeuten, dass zunächst entsprechend vorliegenden Erkenntnissen die Haltungsbedingungen so zu verändern wären, dass die Eingriffe überflüssig werden. Das routinemäßige Kupieren von Schwänzen bei Saugferkeln ist nach geltendem europäischem Recht beispielsweise bereits seit 1994 verboten. Auch 25 Jahre später wird allerdings die Mehrzahl der konventionell gehaltenen Schweine in Europa nach wie vor kupiert. Deutlicher Handlungsdruck entstand aktuell dadurch, dass sich die für den Tierschutz zuständige Generaldirektion der Europäischen Kommission (EU KOM) als Schwerpunktthema für die nächsten Jahre die Durchsetzung der EU-Vorschriften für Tierschutz in der Schweinehaltung vorgenommen hat. Hierbei liegt der Fokus auf der Durchsetzung der Rechtsvorgaben bezüglich des Schwanzkupierens. In den Jahren 2017 und 2018 wurden die Niederlande, Spanien, Dänemark, Italien und Deutschland durch die EU KOM auditiert. Als Ergebnis dieser Auditserien schlussfolgerte die EU KOM, dass in allen untersuchten Ländern routinemäßig kupiert und somit gegen EU-Recht verstoßen wird. ${ }^{3}$ Aufgrund dessen hat die EU KOM 26 Mitgliedsstaaten aufgefordert, einen Aktionsplan vorzulegen, in dem die geplanten Maßnahmen zur Verbesserung der amtlichen Kontrollen bezüglich der Verringerung der Notwendigkeit des Schwanzkupierens verbindlich festgelegt werden. Die EU-Kommission behält sich das Recht vor, alle erforderlichen Maßnahmen zu ergreifen, um sicherzustellen, dass die Mitgliedsstaaten die entsprechenden Rechtsvorgaben umsetzen. Das Beispiel macht im Wesentlichen Folgendes deutlich:

1. Tierschutzvorschriften werden in zentralen Bereichen über sehr lange Zeiträume nicht umgesetzt,

2. es fehlen offenbar Überwachungsmechanismen und/oder die Bereitschaft zur Einhaltung von Rechtsvorschriften und

3. Politik und Praxis ignorieren Rechtsvorschriften bis zur Androhung von Strafen durch die zuständigen EU-Kommissionen.

3 https:/www.laves.niedersachsen.de/startseite/tiere/tierschutz/tierhaltung/schweine/ nationaler-aktionsplan-zur-verbesserung-der-kontrollen-zur-verringerung-der-notw endigkeit-des-schwanzkupierens-bei-schweinen-174707.html (abgerufen am 08.04.2020). 


\subsection{Bestandsgrößenentwicklung und Tierwohl}

Im Widerspruch zur „Tiergerechtheit“ steht in der öffentlichen Wahrnehmung das Bestandsgrößenwachstum tierhaltender Betriebe. Dieses erfolgte und erfolgt im Wesentlichen zur Steigerung der Ressourceneffizienz. Einem kontinuierlichen Rückgang der landwirtschaftlichen Betriebe steht wie eingangs beschrieben ein stetiges Größenwachstum entgegen. Das Bestandsgrößenwachstum hat z. B. bei rinderhaltenden Betrieben neben dem vermehrten Einsatz von Fremdarbeitskräften zu einer zunehmenden Automatisierung, u. a. im Bereich der Fütterung oder des Melkens bei Kühen, geführt. Während zahlreiche Untersuchungen zu den Zusammenhängen zwischen Managementfaktoren sowie verschiedenen Umweltparametern auf Primärmerkmale vorliegen, gibt es kaum Arbeiten zur Korrelation von Betriebs- oder Gruppengrößen und dem Wohlbefinden, der Leistung bei Tieren sowie der Ressourceneffizienz. Sophie Meyer-Hamme (2015) stellt fest, dass auch im Bereich der Mastschweinehaltung Kenntnisse zu Fragen der Zusammenhänge von Tierwohlkriterien und Bestandsgröße sowohl auf theoretischer wie auch anhand empirischer Daten gering sind. Gleichwohl liegen für verschiedene Aspekte einzelne Ergebnisse vor. So fanden z. B. Karin Alvåsen et al. (2012) steigende Mortalitäten in Abhängigkeit von der Herdengröße bei Milchkühen in Schweden. Vergleichbare Effekte fanden Fritha M. Langford et al. (2009) dagegen nicht. Positive Effekte der Gruppengröße auf die Inzidenzrate von Mastitiden beim Milchrind fanden dagegen z. B. Marie J. Haskell et al. (2009). Auch Daniel Gieseke et al. (2015) fanden bei Untersuchungen zum Tierwohl in größeren Milchviehbeständen sogar im Durchschnitt bessere Ergebnisse.

Sophie Meyer-Hamme (2015) führte erstmals systematische Untersuchungen zu Fragen der Zusammenhänge von Tierwohlkriterien und Bestandsgröße in niedersächsischen Mastschweinebetrieben mittels eines tierorientierten Indikatorensystems nach dem Welfare Quality® Protokoll (WQP) durch. Die Ergebnisse zeigen, dass die Bestandsgröße zumindest in konventionellen Betrieben als Indikator für unzureichendes Wohlergehen der Tiere nicht geeignet ist. D. h., dass eine hohe Anzahl an Mastschweinen pro Bestand nicht automatisch auf ein niedriges Tierwohlniveau hinweist. Allerdings stellte die Autorin fest, dass das Tierwohlniveau in den untersuchten Betrieben insgesamt als eher niedrig einzustufen ist, was möglicherweise das Ergebnis beeinflusst hat. 


\section{Voraussetzungen für positive Veränderungen in der Nutztierhaltung}

Die Nutztierhaltung hat sich in einigen Ländern Europas zweifelsohne in den letzten Jahrzehnten zu einem wirtschaftlich sehr erfolgreichen Sektor entwickelt. Gleichzeitig gibt es allerdings erhebliche Defizite vor allem im Bereich des Tierwohls sowie des Umweltschutzes. In Kombination mit einer veränderten Einstellung zur Mensch-Tier-Beziehung, die die Verantwortung des Menschen für das Tier stärker in den Vordergrund rückt, führte dies zu einer verringerten gesellschaftlichen Akzeptanz der Nutztierhaltung (Harald Grethe et al. 2015). Daraus erwächst eine Bedrohung der Existenz der Nutztierhaltung in Europa. Dabei würde eine Abwanderung der Erzeugung vermutlich eher zu einer Verschlechterung der Situation der Nutztiere führen, da die gesetzlichen Standards zur Nutztierhaltung in vielen Bereichen der Welt sogar hinter denen der EU zurückfallen. Der Wissenschaftliche Beirat für Agrarpolitik beim deutschen Bundesministerium für Ernährung und Landwirtschaft (WBA) hat deshalb Leitlinien und Empfehlungen für eine gesellschaftlich akzeptierte Nutztierhaltung entwickelt, die zu einer grundlegenden Veränderung der Nutztierhaltung führen sollen. Der WBA empfahl dafür ein umfangreiches Maßnahmenbündel und zudem einen intensiven Diskurs zwischen Wirtschaft, Zivilgesellschaft und Politik unter Einbeziehung der Wissenschaft.

Der WBA hat die folgenden Leitlinien für eine zukunftsfähige Tierhaltung aus Sicht des Tierschutzes genannt:

1. Zugang aller Nutztiere zu verschiedenen Klimazonen, vorzugsweise Außenklima,

2. Angebot unterschiedlicher Funktionsbereiche mit verschiedenen Bodenbelägen,

3. Angebot von Einrichtungen, Stoffen und Reizen zur artgemäßen Beschäftigung, Nahrungsaufnahme und Körperpflege,

4. Angebot von ausreichend Platz,

5. Verzicht auf Amputationen,

6. routinemäßige betriebliche Eigenkontrollen anhand tierbezogener Tierwohlindikatoren,

7. deutlich reduzierter (bzw. optimierter) Arzneimitteleinsatz,

8. verbesserter Bildungs-, Kenntnis- und Motivationsstand der im Tierbereich arbeitenden Personen und

9. stärkere Berücksichtigung funktionaler Merkmale in der Zucht (WBA 2015, ii).

Einige Punkte haben Wissenschaft und Praxis bereits in den letzten Jahren verstärkt aufgegriffen, andere müssen noch stärker berücksichtigt werden. 
Für viele Nutztierarten sind die genannten Punkte vergleichsweise leicht und kostenneutral realisierbar, andere stellen dagegen erhebliche Herausforderungen für bestimmte Bereiche dar und führen zu Kostensteigerungen. Dementsprechend müssen Maßnahmen ergriffen werden, die sicherstellen, dass es zu keiner einseitigen Belastung der Landwirtschaft kommt und damit Produktionsanteile ins nichteuropäische Ausland abwandern, vor allem dorthin, wo Tierschutzstandards unter denen der EU liegen (Harald Grethe et al. 2015). Insgesamt gesehen zielt das Gutachten auf einen Zustand ab, der in etwa 15 bis 20 Jahren erreicht werden soll. Ein vergleichbar umfassendes Gutachten zu Perspektiven der Nutztierhaltung in Deutschland gab es zuvor nicht. Entsprechend hat es teilweise zu sehr heftigen Reaktionen geführt. ${ }^{4}$ Es hat aber auch dazu geführt, dass sich die Politik wesentlich intensiver des Themas angenommen hat und die Diskussionen um die Zukunft der Nutztierhaltung in Deutschland und Europa weiter anhalten und Schritt für Schritt zur Verbesserung des Tierwohlniveaus führen (u. a. durch verbesserte Kontrollen zur Einhaltung gesetzlicher Mindestvorgaben). Dies ist aus der Verantwortung des Menschen für das Tier auch zwingend notwendig.

Im Jahr 2019 hat die deutsche Bundesministerin für Ernährung und Landwirtschaft Julia Klöckner ein „Kompetenznetzwerk Nutztierhaltung“ eingesetzt. Unter dem Vorsitz des ehemaligen Bundeslandwirtschaftsministers Jochen Borchert und der Mitarbeit von Entscheidungsträgern und Fachleuten aus Politik, Wissenschaft, Praxis, Wirtschaft sowie Verbänden sollte eine Bewertung und Darstellung möglicher Entwicklungen der Nutztierhaltung erfolgen. Die Kommission sollte die aktuellen Entwicklungen und Herausforderungen aus allen Bereichen der Nutztierhaltung analysieren, Lösungswege für den Umbau der Nutztierhaltung in Deutschland erarbeiten sowie Ansätze für die Verbesserung der gesellschaftlichen Akzeptanz der Nutztierhaltung in Deutschland aufzeigen und mögliche Finanzierungsmodelle durchdenken. Die Kommission bestätigt in ihren Empfehlungen vom 11. Februar 2020 das Gutachten des WBA dahingehend, dass auch sie die Nutztierhaltung in Deutschland in ihrer gegenwärtigen Form als nicht zukunftsfähig bewertet. Deshalb empfiehlt auch das Kompetenznetzwerk einen grundlegenden und langfristigen Umbau der deutschen Nutztierhaltung - hin zu mehr Tierwohl und Umweltschutz.

4 Siehe u. a. die Antwort der Bundesregierung auf die Kleine Anfrage der Abgeordneten Friedrich Ostendorff, Nicole Maisch, Harald Ebner und der Fraktion der BÜNDNIS 90/DIE GRÜNEN - Drucksache 18/6140 - Umsetzung der Empfehlungen des Wissenschaftlichen Beirats für Agrarpolitik durch die Bundesregierung, in: http://dipbt.bundestag.de/doc/btd/18/062/1806293.pdf (abgerufen am 13.04.2020). 
Das soll nach deren Vorstellungen schrittweise und tierartspezifisch über einen sehr langen Zeitraum bis 2040 erfolgen. Die Umstellung der Haltungssysteme soll dabei stufenweise erfolgen und jeweils mit einer Erhöhung des Tierwohlstandards (begleitet von der Einführung freiwilliger und staatlicher Tierwohllabels) einhergehen. Die Kommission beziffert die Kosten auf anfangs 1,2 und später 3,6 Milliarden Euro jährlich. Erfolgen keine Veränderungen, sieht auch diese Kommission aufgrund einer stetig sinkenden Akzeptanz der Bevölkerung die Abwanderung der Erzeugung ins nicht europäische Ausland als Konsequenz.

\section{Literatur}

Alvåsen, Karin/Jansson Mörk, Marie/Hallén Sandgren, Charlotte/Thomsen, Peter Thorup et al. 2012, Herd-level risk factors associated with cow mortality in Swedish dairy herds, in: Journal of Dairy Science 95, 4352-4362.

von Alvensleben, Reimar 2003, Wie sieht der Verbraucher die Nutztierhaltung? Analysen - Konsequenzen - Perspektiven, in: Nutztierpraxis 4, 50-55.

Antonovsky, Aaron 1996, The salutogenic model as a theory to guide health promotion, in: Health Promotion International 11, 11-18.

Armbrecht, Linda/Lambertz, Christian/Albers, Dirk/Gauly, Matthias 2015, Tierwohl von Milchkühen bei Stall- und Weidehaltung - Ein Vergleich anhand des Welfare Quality® Protokolls, in: Gieseke, Daniel et al. (Hg.) 2015, Tierhaltung im Spannungsfeld von Tierwohl, Ökonomie und Gesellschaft (Tagungsband zur Tierwohl-Tagung 2015 in Göttingen), Göttingen, 70-72.

Boissy, Alain/Manteuffel, Gerhard/Jensen, Margit Bak/Oppermann Moe, Randi et al. 2007, Assessment of positive emotions in animals to improve their welfare, in: Physiology \& Behavior 92 (3), 375-397.

Busch, Gesa/Kayser, Maike/Spiller, Achim 2013, „Massentierhaltung“ aus VerbraucherInnensicht - Assoziationen und Einstellungen, in: Jahrbuch der österreichischen Gesellschaft für Agrarökonomie 22 (1), 61-70.

Destatis - Deutsches Statistisches Bundesamt 2011, Wer produziert unsere Nahrungsmittel? Aktuelle Ergebnisse der Landwirtschaftszählung 2010, Wiesbaden, in: https://www.destatis.de/GPStatistik/servlets/MCRFileNodeServlet/DEMonog rafie_derivate_00001484/WerProduziertNahrungsmittel.pdf (abgerufen am 13.04.2020).

Destatis - Deutsches Statistisches Bundesamt 2020, Land- und Forstwirtschaft, Fischerei - Tiere und tierische Erzeugung, in: https://www.destatis.de/DE/Themen /Branchen-Unternehmen/Landwirtschaft-Forstwirtschaft-Fischerei/Tiere-Tierisc he-Erzeugung/_inhalt.html (abgerufen am 17.04.2020).

DLG (Hg.) 2009, Landwirtschaft 2020 - Herausforderungen, Strategien, Verantwortung (Archiv der DLG 103), Frankfurt a. M. 
DLG (Hg.) 2010, Zukunftsbranche Landwirtschaft. Unternehmerisch - innovativ verantwortungsvoll (Archiv der DLG 104), Frankfurt a. M.

DMK - Deutsches Milchkontor GmbH 2013, Nachhaltigkeitsprogramm DMK 2020: Unsere Milch. Unsere Welt. Kurzbericht 2014/2015, Zeven.

Gauly, Matthias 2015, Akzeptanz der Nutztierhaltung: Analyse aus der Sicht der Agrarwissenschaften, in: Akzeptanz der Nutztierhaltung - Herausforderungen im Bereich Futter und Fütterung (Tagungsband der 53. Jahrestagung der Bayerischen Arbeitsgemeinschaft Tierernährung e. V.), Freising, 10-16.

Gieseke, Daniel/Lambertz, Christian/Traulsen, Imke/Gauly, Matthias et al. 2014, Beurteilung von Tiergerechtheit in der Milchviehhaltung - Evaluierung des Welfare Quality® Protokolls für Rinder, in: Züchtungskunde 86, 58-70.

Gieseke, Daniel/Lambertz, Christian/Gauly, Matthias 2015, Besteht ein Zusammenhang zwischen Bestandsgröße und Tierwohl in der konventionellen Milchviehhaltung? Beitrag bei der Tierwohl-Tagung in Göttingen, 07.-08.10.2015, in: https://www.researchgate.net/publication/286443379 (abgerufen am 13.04.2020).

Grethe, Harald/Christen, Olaf/Balmann, Alfons/Gauly, Matthias et al. 2015, Wege zu einer gesellschaftlich akzeptierten Nutztierhaltung. Gutachten Wissenschaftlicher Beirat für Agrarpolitik beim Bundesministerium für Ernährung und Landwirtschaft (Berichte über Landwirtschaft, Sonderheft 221), Berlin.

Haskell, Marie J./Langford, Fritha M./Jack, Mhairi C./Sherwood, Lorna et al. 2009, The effect of organic status and management practices on somatic cell counts on UK dairy farms, in: Journal of Dairy Science 92, 3775-3780.

Knierim, Ute 2001, Grundsätzliche ethologische Überlegungen zur Beurteilung der Tiergerechtheit bei Nutztieren, in: Deutsche Tierärztliche Wochenschrift 109, 261-266.

Knierim, Ute 2010, Ansätze aus dem EU-Projekt Welfare Quality® zur Beurteilung des Wohlergehens landwirtschaftlicher Nutztiere, in: Aktuelle Probleme des Tierschutzes. 30. Fortbildungsveranstaltung der ATF-Fachgruppe Tierschutz des Institutes für Tierhygiene, Tierschutz und Nutztierethologie, Hannover, 16.-17.09.2010: Vorträge und Kurzfassungen, 7-12.

Kühl, Sarah/Ermann, Manuel/Spiller, Achim 2014, Imageträger Weidegang, in: DLG-Mitteilungen Heft 4/2014, 94-97.

Langford, Fritha M./Rutherford, Kenneth M./Jack, Mhairi C./Sherwood, Lorna et al. 2009, A comparison of management practices, farmer-perceived disease incidence and winter housing on organic and non-organic dairy farms in the UK, in: Journal of Dairy Research 76, 6-14.

Lassen, Birthe/Lindena, Tomke/Claus, Anna Sophie 2019, 5000 Milchbauern im Nachhaltigkeits-Check, in: Top Agrar 48 (2), R6-R8.

Meyer-Hamme, Sophie 2015, Zusammenhang zwischen Bestands-, Gruppengröße und Indikatoren des Tierwohls in der konventionellen Schweinemast. Dissertation. Fakultät für Agrarwissenschaften, Universität Göttingen. 
Meyer-Hamme, Sophie/Lambertz, Christian/Gauly, Matthias 2016, Does group size have an impact on welfare indicators in fattening pigs?, in: Animal 10 (1), 142-149; DOI: $10.1017 /$ S1751731115001779.

Reijs, Joan W./Daatselaar, Co H. G./Helming, John F. M./Jager, Jakob H. et al. 2013, Grazing dairy cows in North-West Europe. Economic farm performance and future developments with emphasis on the Dutch situation (LEI Report 2013-001), Wageningen.

Roffeis, Margret/Waurich, Benno 2013, Hohe Milchleistungen und gesunde Euter. Ergebnisse aus den RBB-Testherden. Unveröffentlichter Beitrag beim Milchrindtag der Rinderunion Berlin-Brandenburg, Götz, 10.01.2013.

Römer, Anke 2011, Untersuchungen zur Nutzungsdauer bei Deutschen Holstein Kühen, in: Züchtungskunde 83, 8-20.

Sachverständigenrat für Umweltfragen 2015, Stickstoff: Lösungsstrategien für ein drängendes Umweltproblem. Sondergutachten, Berlin.

Sandilands, Victoria 2011, The laying hen and bone fractures, in: The Veterinary Record 169 (16), 411-412.

Smidt, Diedrich 1996, Gesunde Tiere - Grundlage einer verantwortungsbewussten und wirtschaftlichen Tierhaltung, in: Tierärztliche Umschau 51, 519-523.

Sonoda Lilia Thays/Fels, Michaela/Oczak, Maciej/Vranken, Erik et al. 2013, Tail biting in pigs - causes and management intervention strategies to reduce the behavioural disorder. A review, in: Berliner und Münchener Tierärztliche Wochenschrift 126, 104-112.

Theuvsen, Ludwig/Deimel, Mark 2011, Informationsaustausch in Wertschöpfungsketten der Veredelungswirtschaft: Ein Beitrag zur Verbesserung der Zukunftsfähigkeit räumlicher Verbundsysteme, in: Windhorst, Hans-Wilhelm/Veauthier, Aline (Hg.) 2011, Nachhaltige Tierproduktion in agrarischen Intensivgebieten Niedersachsens, Vechta, 13-32.

Thomet, Peter/Piccand, Valérie 2011, Ressourceneffiziente Milchproduktion Welcher Kuhtyp ist geeignet?, in: 38. Viehwirtschaftliche Fachtagung, 13.-14.04.2011, Bericht LFZ, Raumberg-Gumpenstein, 11-18.

Tierschutzgesetz in der Neufassung vom 18.05.2006 (BGBl. I Nr.25 vom 31.05.2006 S. 1206), zuletzt geändert durch Gesetz vom 04.07.2013 (BGBl. I von 12.07.2013 S. 2182).

Tierschutz-Nutztierhaltungsverordnung (Verordnung zum Schutz landwirtschaftlicher Nutztiere und anderer zur Erzeugung tierischer Produkte gehaltener Tiere bei ihrer Haltung - TierSchNutztV) vom 22.08.2006 (BGBl. I S. 2043), zuletzt geändert durch Verordnung vom 05.02.2014 (BGBl. I S. 94).

VIT - Vereinigte Informationssysteme Tierhaltung w. V. (Verden), Jahresberichte 2004 bis 2013.

WBA - Wissenschaftlicher Beirat für Agrarpolitik beim deutschen Bundesministerium für Ernährung und Landwirtschaft 2015, Wege zu einer gesellschaftlich akzeptierten Nutztierhaltung. Gutachten, Berlin. 
Welfare Quality 2009, Welfare Quality ${ }^{\circledR}$ assessment protocol for pigs (sow and piglets, growing and finishing pigs). Welfare Quality® Consortium. Lelystad, Netherlands.

ZMP - Zentrale Markt- und Preisberichtstelle für Erzeugnisse der Land-, Forstund Ernährungswirtschaft $\mathrm{GmbH}$, seit 2011 Zentrale Markt- und Preisinformationen $\mathrm{GmbH}$ (Bonn), Jahresberichte 2003 und 2012. 